\title{
Bone-muscle crosstalk between osteocytes and skeletal muscle through microRNA21 removal leads to sex dependent increase in muscle mass
}

Andrew Sickbert ${ }^{1}$, Dr. Lillian Plotkin ${ }^{2}$

${ }^{1}$ Indiana University School of Medicine, ${ }^{2}$ Indiana University School of Medicine, Department of Anatomy and Cell Biology

Background and Hypothesis: Previous studies suggested that microRNA removal from osteocytes, the cells embedded in the bone matrix, not only has effects on bone, but also increases muscle mass only in female mice. This suggested that changes in bone cells alters skeletal muscle through bone-muscle crosstalk. We therefore tested the hypothesis that osteocytic microRNA21 removal leads to release of factors that can influence skeletal muscle.

Experimental Design or Project Methods: Osteocytic microRNA21-deficient mice were generated by mating microRNA2 $1^{\text {floxflox }}$ mice with mice expressing Cre recombinase in osteocytes. Lean body mass was measured in 4-month-old male and female mice by DXA/Piximus. Femurs/tibias were isolated and cultured in the presence of growing media for $48 \mathrm{~h}$. Media was collected (CM) and transferred to differentiating $\mathrm{C} 2 \mathrm{C} 12$ myoblastic cells. After $48 \mathrm{~h}$, cells were fixed and stained for myosin heavy chain. Myotube diameter was measured using ImageJ software. RNA was extracted from gastrocnemius muscle using Trizol, and gene expression was measured by qPCR. Statistical analysis was performed by t-test within each sex.

Results: Percent lean body mass was $3.33 \%$ higher in female microRNA21 knockouts compared to female microRNA2 $1^{\text {flox/flox }}$ (control) mice, with no changes observed in males. Further, CM from female knockout mice increased the mean myotube diameter by $24.3 \%$ compared to control females. However, we did not find any differences in the levels of expression of skeletal muscle genes in females or males.

Conclusion and Potential Impact: We conclude that microRNA21 deletion from osteocytes in female mice results in the release of factor(s) with anabolic or anticatabolic effects in skeletal muscle. As we determined that osteocytes must be releasing factors that influences muscle growth, future studies can be directed at specifically finding which osteocyte cytokines and/or genes are directly involved in muscle homeostasis, and whether microRNA deletion also affects skeletal muscle function/strength. 